\title{
A Uniform Series of Low-Latitude Coronal Holes in 1973-2018
}

\author{
A. Hamada ${ }^{1}$ (D) T. Asikainen ${ }^{1}$ K. Kursula ${ }^{1}$
}

Received: 14 July 2020 / Accepted: 5 February 2021 / Published online: 24 February 2021

(C) The Author(s) 2021

\begin{abstract}
Coronal holes (CHs) are regions in the solar corona characterized by plasma density lower than in the surrounding quiet Sun. Therefore they appear dark in images of the solar atmosphere made, e.g., in extreme ultraviolet (EUV). Identifying CHs on solar images is difficult since $\mathrm{CH}$ boundaries are not sharp, but typically obscured by magnetic structures of surrounding active regions. Moreover, the areas, shapes, and intensities of CHs appear differently in different wavelengths. Coronal holes have been identified both visually by experienced observers and, more recently, by automated detection methods using different techniques. In this article, we apply a recent, robust $\mathrm{CH}$ identification algorithm to a new set of homogenized EUV synoptic maps based on four EUV lines measured by the Solar and Heliospheric Observatory/Extreme ultraviolet Imaging Telescope (SOHO/EIT) in 1996-2018 and the Solar Dynamics Observatory/Atmospheric Imaging Assembly (SDO/AIA) in 20102018 and create corresponding $\mathrm{CH}$ synoptic maps. We also use $\mathrm{CHs}$ of the hand-drawn McIntosh archive (McA) from 1973-2009 to extend the CH database to earlier times. We discuss the success of the four EUV lines to find $\mathrm{CHs}$ at high or low latitudes, and confirm that the combined EIT $195 \AA$ A/AIA $193 \AA$ series applies best for both polar and low-latitude $\mathrm{CH}$ detection. While the polar $\mathrm{CH}$ detection suffers from the vantage-point limitation, the low-latitude $\mathrm{CH}$ areas extracted from this line correlate with the McA $\mathrm{CH}$ data very well. Using the simultaneous measurements between EIT and McA and EIT and AIA, we scale the different data series to the same level and form the longest uniform series of low-latitude $\mathrm{CHs}$ in 1973-2018. We find that, while the solar cycle maxima of low-latitude CHs in the descending phase of Solar Cycles 21-23 attain roughly similar values, the corresponding maximum during Solar Cycle 24 is reduced by a factor of two. This suggests that magnetic flux emergence is crucial for the formation of low-latitude CHs.
\end{abstract}

A. Hamada

amr.hamada@oulu.fi

$\bowtie$ T. Asikainen

timo.Asikainen@oulu.fi

$凶$ K. Mursula

Kalevi.Mursula@oulu.fi

1 Space Climate Research Group, Space Physics and Astronomy Research Unit, University of Oulu, Oulu, Finland 
Keywords Coronal holes · Solar observation · Dataset · Solar cycle

\section{Introduction}

Coronal holes $(\mathrm{CHs})$ are regions characterized by a lower electron density $\left(\approx 4 \times 10^{8} \mathrm{~cm}^{-3}\right)$ than in the typical quiet Sun $(\mathrm{QS})\left(\approx 1.6 \times 10^{9} \mathrm{~cm}^{-3}\right.$; Phillips, 1995), and therefore typically appear darker in extreme ultraviolet (EUV) images than the surrounding quiet Sun. Coronal holes were first observed by Waldmeier (1957) who noticed long-lived regions of negligible intensity in coronagraphic images of the $5303 \AA$ green line emission. The earliest extreme ultraviolet (EUV) observations of CHs were made by Tousey, Sandlin, and Purcell (1968), who studied spectroheliograms obtained by rocket experiments and noted that EUV emission in polar regions seemed to be weaker than in the surrounding regions.

Coronal holes were first identified visually, tracing the perceived $\mathrm{CH}$ boundaries by hand (Harvey and Recely, 2002). However, different observers typically determine the $\mathrm{CH}$ boundaries differently. Initial research in the segmentation of $\mathrm{CHs}$ involved hand-drawn synoptic maps of $10830 \AA$ He I images (Harvey and Recely, 2002). Other approaches to CH identification were made by Chapman and Bromage (2002) and Chapman (2007) who constructed synoptic $\mathrm{CH}$ maps using measurements made with the Solar and Heliospheric Observatory (SOHO) Coronal Diagnostic Spectrometer (CDS: Harrison (1997)).

More recently, there have been several attempts to automate the identification of CHs using different techniques (e.g. Krista and Gallagher, 2009; Illarionov, Kosovichev, and Tlatov, 2020; Lowder et al., 2014; Caplan, Downs, and Linker, 2016; Heinemann et al., 2019). Automated detection of CHs by intensity thresholding using one wavelength is complicated by the presence of dark quiet-Sun regions, filaments, and transient dimmings which all have similar range of intensities as CHs. Also, many of the algorithms that have been developed for the EUV image segmentation are difficult to implement for $\mathrm{CH}$ detection due to the noisy nature of these images, leading to obscurely defined $\mathrm{CH}$ boundaries, and due to other features of EUV images, such as the rather similar intensities of $\mathrm{CH}$ and dark quiet-Sun regions (Caplan, Downs, and Linker, 2016). To resolve these ambiguities, it is necessary to make use of additional information from other wavelengths, from magnetograms, or from the time evolution of the feature in order to check the consistency of a $\mathrm{CH}$ candidate with actual physical parameters. A recently developed algorithm by Hamada et al. (2018), which we also use in this article, attempts to overcome these problems by detecting $\mathrm{CHs}$ in four different wavelengths and using magnetogram data to ensure that the detected dark $\mathrm{CH}$ candidates have a sufficiently unipolar magnetic field to differentiate them from dark filament channels which do not have a preferred net magnetic polarity.

Hamada, Asikainen, and Mursula (2019) have recently constructed a new, homogenized set of EUV synoptic maps from the Solar and Heliospheric Observatory/Extreme ultraviolet Imaging Telescope (SOHO/EIT) and the Solar Dynamics Observatory/Atmospheric Imaging Assembly (SDO/AIA) measurements extending from 1996 and 2010 to 2018, respectively. These synoptic maps have been constructed using the same spatial resolution, which reduces the differences between the maps that have different resolutions. The maps are presented in a standardized logarithmic intensity scale to avoid problems due to significant drifts in the SOHO/EIT and SDO/AIA intensities. Also, all EIT pixel values were scaled to the AIA level. Here we use this dataset to construct a new synoptic dataset of CHs (19962018). Many previous studies (Kirk et al., 2009; Krista and Gallagher, 2009; de Toma, 2011; Tlatov, Tavastsherna, and Vasil'eva, 2014; Hess Webber et al., 2014; Karna, Hess Webber, 
Table 1 Selected SOHO/EIT and SDO/AIA wavelengths.

\begin{tabular}{lllll}
\hline Source & Wavelength & Ion & $\begin{array}{l}\text { Peak temperature } \\
(\mathrm{MK})\end{array}$ & Region \\
\hline SOHO/EIT & $284 \AA$ & Fe XV & 2.0 & Outer corona \\
SDO/AIA & $211 \AA$ & Fe XIV & 2.0 & Outer corona \\
SOHO/EIT & $195 \AA$ & Fe XII & 1.6 & Corona \\
SDO/AIA & $193 \AA$ & Fe XII & 1.6 & Corona \\
SOHO/EIT and SDO/AIA & $171 \AA$ & Fe IX-X & 1.3 & Transition region \\
SOHO/EIT and SDO/AIA & $304 \AA$ & He II & 0.08 & Chromosphere \\
\hline
\end{tabular}

and Pesnell, 2014; Lowder, Jiong, and Leamon, 2017) have considered the long-term evolution of $\mathrm{CHs}$ through different phases of the solar cycle. However, these studies have considered only one or two solar cycles. A longer record of low-latitude $\mathrm{CHs}$ developed here spans over more than four full solar cycles, giving a broader context to the coronal observations made during Solar Cycles 23 and 24. Moreover, there are indications that the photospheric and coronal magnetic structure have suddenly and systematically changed during this time period (e.g. Lukianova and Mursula, 2011; Virtanen, Koskela, and Mursula, 2020).

In Section 2 we discuss the construction of new $\mathrm{CH}$ synoptic maps from the EUV synoptic maps using the algorithm developed by Hamada et al. (2018), with certain modifications. Section 3 includes the analysis of the long-term dataset of hand-traced $\mathrm{CH}$ synoptic maps of the McIntosh Archive (McA). In Section 4 we compare the new $\mathrm{CH}$ synoptic maps to the ones extracted from McA dataset, showing the spatial-temporal evolution of $\mathrm{CHs}$ in the different datasets. Altogether these datasets provide measurements of CHs from 1973 to present. Section 5 shows the latitudinal variation of the correlation between the $\mathrm{CH}$ areas from the different observations. We discuss how the CHs based on different EUV wavelengths compare with the McA maps and with each other at different heliographic latitudes. In Section 6 we compare the correlation of CHs based on McA and the 195/193 A EUV wavelengths at different latitude bands and construct a uniform dataset of low-latitude CHs from $-60^{\circ}$ to $+60^{\circ}$ from 1973 to 2018 . The results of the study are summarized in Section 7 .

\section{Coronal Hole Dataset Based on SOHO/EIT and SDO/AIA CH Synoptic Maps}

Hamada, Asikainen, and Mursula (2019) recently constructed a new homogeneous database of synoptic EUV maps from SOHO/EIT and SDO/AIA full-disk images (http://satdat.oulu. fi/solar_data/). These synoptic maps were constructed for four wavelengths of both instruments. The characteristics of these four wavelengths are summarized in Table 1 (see also Moses et al., 1997; Petkaki et al., 2012). These synoptic maps have been constructed by concatenating $13.3^{\circ}$ wide central solar meridian strips (corresponding to 1 image/day) for both instruments. The longitude of a strip in the synoptic map is determined by computing the Carrington longitude of the central meridian during the time of the corresponding full-disk image. The synoptic EUV maps of this database were standardized and the EIT based maps were statistically transformed so that the average standardized intensity distributions of EIT images match those of AIA images during the time when simultaneous images from both instruments are available. These homogenized EUV maps describe relative logarithmic intensity variations within each map. The homogenization procedure removes 
Table 2 Data coverage of standardized and homogenized synoptic maps available for the instruments used. Dates refer to the start and end times of Carrington rotations.

\begin{tabular}{lllllll}
\hline Source & Observable & \multicolumn{2}{l}{ Start } & & \multicolumn{2}{l}{ End } \\
& & CR & Date & & CR & Date \\
\hline SOHO/EIT & $284 \AA, 195 \AA, 171 \AA, 304 \AA$ & 1906 & 13.02 .1996 & & 2211 & 23.11 .2018 \\
SDO/AIA & $211 \AA, 193 \AA, 171 \AA, 304 \AA$ & 2097 & 20.05 .2010 & & 2211 & 23.11 .2018 \\
KPNO/He I & $10830 \AA$ & 1601 & 05.05 .1973 & & 2086 & 23.07 .2009 \\
\hline
\end{tabular}

temporal changes of image average intensity caused, e.g. by solar cycle (SC) variation, or by the degradation of EIT and AIA sensors. The homogenized maps produced in this way are well suited for studying CHs (Hamada, Asikainen, and Mursula, 2019). The SOHO/EIT and SDO/AIA based synoptic EUV maps span from 1996 to 2018 and from 2010 to 2018, respectively (see Table 2 ).

We use the image segmentation algorithm developed by Hamada et al. (2018) to determine the intensity threshold for $\mathrm{CHs}$, so that pixels darker than the threshold are identified as $\mathrm{CH}$ pixels. The algorithm determines the $\mathrm{CH}$ threshold intensity for each map separately by finding optimal segments, which best show a bimodal distribution of pixel intensities: the darker population of $\mathrm{CHs}$ and the brighter population of the quiet Sun. The algorithm finds the intensity thresholds for optimal segments of 11 different square-formed segment sizes (from $15 \times 15$ to $45 \times 45$ in three pixel steps). The mean and standard deviation of these optimal thresholds provides an estimate of the $\mathrm{CH}$ intensity threshold for the respective synoptic map as well as its uncertainty. Figure 1 shows the EIT/195 ̊ synoptic map for Carrington rotation (CR) 2184 with ten optimal segments denoted in magenta and one optimal segment (for $35 \times 35$ pixel size) denoted in yellow. The yellow segment histogram shows a bimodal distribution, which is not seen in the histogram of the whole synoptic map. The $\mathrm{CH}$ threshold intensity for all 11 optimal segments is determined as the intersection between these two distributions.

The intensity threshold and its uncertainty were determined in this way for all SOHO/EIT and SDO/AIA synoptic maps of the four wavelengths of both instruments. Note that, although there are subtle differences in the temperature response functions of the AIA/193 $\AA$, and EIT/195 Å channels, they are similar enough to be used together to generate synchronic maps especially with proper preprocessing (Caplan, Downs, and Linker, 2016). Figure 2 shows the temporal variation of the $\mathrm{CH}$ threshold intensities with their uncertainties for four EIT and AIA wavelengths. Note that the threshold intensities of the 193/195 $\AA$ and $304 \AA$ wavelengths do not display notable systematic evolution. There is some variation in the threshold intensities of these wavelengths but it is rather random and mostly within the uncertainty. Accordingly, we find quite constant threshold intensity estimates for these two wavelengths.

For EIT/284 A, AIA/211 A, and EIT/AIA $171 \AA$ wavelengths, Figure 2 shows quite a clear SC variation in the threshold intensity. For $284 / 211 \AA$ it is in anti-phase and for $171 \AA$ in phase with the sunspot cycle. The out-of-phase behavior is most likely caused by the SC variation of active regions, which have enhanced EUV intensities and whose number and area maximize during solar maxima.

The smoothed threshold intensities (constant values for 193/195 $\AA$ and $304 \AA$, and 31CR running-mean smoothed threshold for $171 \AA$ and $284 \AA / 211 \AA$ ) were then used for each synoptic map to determine binary maps of $\mathrm{CHs}$ according to the algorithm described by Hamada et al. (2018). Pixels darker than the threshold are defined as $\mathrm{CH}$ pixels. Also, we 

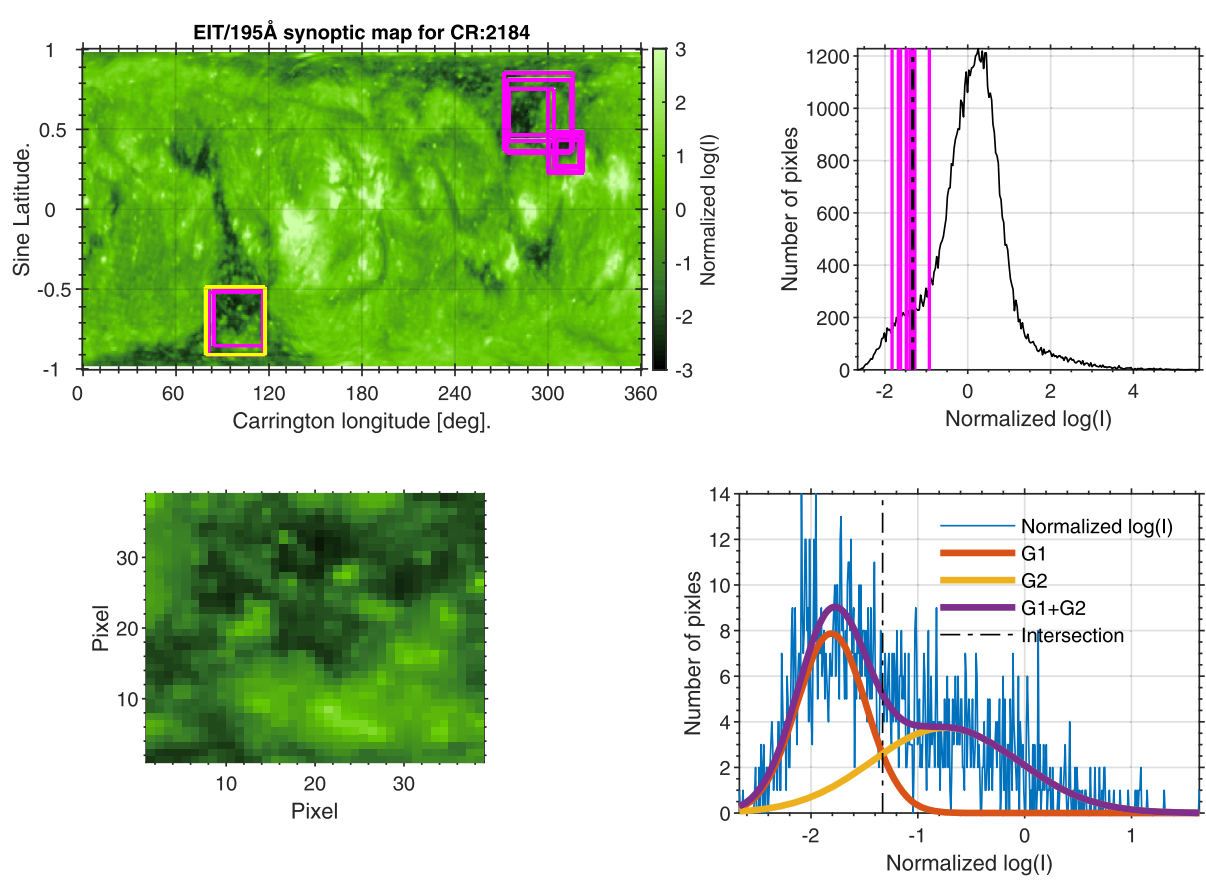

Figure 1 EIT $195 \AA$ A synoptic map for CR2184 (16 November 2016) and its intensity distribution (upper panels). The magenta boxes and one yellow box indicate the locations for the 11 optimal segments of different sizes (yellow $35 \times 35$ ). An expanded view of the yellow box and its intensity histogram are shown in the lower panels. Segment histogram (lower right panel) was fit to a sum of two Gaussians (G1 and G2). Vertical dash-dotted line indicates the $\mathrm{CH}$ threshold intensity.

used the relative magnetic flux imbalance $\left(\left\langle B_{r}\right\rangle /\left\langle\left|B_{r}\right|\right\rangle\right)$ of the radial magnetic field, $B_{r}$, within the initial $\mathrm{CH}$ region to exclude all the filaments from the final $\mathrm{CH}$ regions (Scholl and Habbal, 2008; Hamada et al., 2018). The resulting $\mathrm{CH}$ regions were then simplified using morphological image processing operations in order to remove very small patches of $\mathrm{CHs}$ and very small, separate non- $\mathrm{CH}$ regions inside large $\mathrm{CHs}$ (for details, see Hamada et al. 2018). It should be noted that $\mathrm{CHs}$ often depict high-resolution structure, e.g. small patches of non-CH regions inside larger CHs (e.g. Lowder et al., 2014). The morphological operations employed here (as well as other resolution scaling or smoothing operations) therefore influence the estimated fractional $\mathrm{CH}$ areas. However, since here the $\mathrm{CH}$ s extracted from EUV synoptic maps are compared to hand-drawn $\mathrm{CH}$ maps of much lower effective resolution (see Section 3), the simplifying morphological operations improve the correspondence between the different datasets. This is also the case for comparisons between EIT and AIA, where the same $\mathrm{CH}$ can have somewhat different high-resolution boundaries (e.g. Lowder et al., 2014).

Figures 3 and 4 show the result of automated $\mathrm{CH}$ identification for CR2184 (the same CR used in Figure 1) from SOHO/EIT and SDO/AIA homogenized synoptic maps, respectively. The He II $304 \AA$ images are dominated by emission from structures of the upper chromosphere/transition region and prominences on the solar limb (Benevolenskaya, Kosovichev, and Scherrer, 2001; Liewer, Qiu, and Lindsey, 2017). The Fe XI/Fe X 171 A images display background emission that is present over most of the quiet Sun, while the most intense emission comes from active regions. Unfortunately, in this wavelength the CHs and 


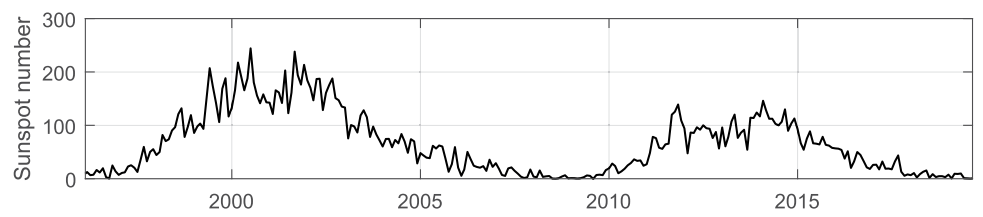

EIT/284 A and AIA/211 A



EIT/195 A and AIA/193 A

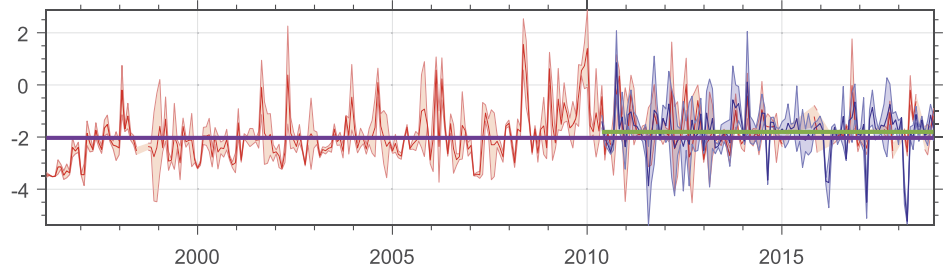

EIT/171 A and AIA/171 A



EIT/304 A and AIA/304 A



Figure $2 \mathrm{CH}$ threshold intensities in standardized logarithmic scale and their errors for (red) SOHO/EIT $284 \AA, 195 \AA, 171 \AA, 304 \AA$ and (blue) SDO/AIA $211 \AA, 193 \AA, 171 \AA, 304 \AA$. The purple and green lines (constructed for $195 \AA, 193 \AA$, and $304 \AA$ lines, 31-CR running-mean values) indicate the final smoothed threshold values used for $\mathrm{CH}$ identification.

filament channels often appear almost equally dark, leading to an excessive amount of erroneously identified CHs. Obviously, this wavelength is not very well suited to determine $\mathrm{CH}$ boundaries (Madjarska and Wiegelmann, 2009). The Fe XII $195 \AA$ and $193 \AA$ images show the inner solar corona with quite separate, contiguous regions and different distributions of 
intensities for $\mathrm{CHs}$ and the quiet Sun. The same is more or less valid for the EIT $284 \AA$ and AIA $211 \AA$, which observe the upper corona at the highest temperature. Figures 3 and 4 (second column panels) show the extracted $\mathrm{CH}$ areas as white regions. Using the solar magnetic synoptic maps (SOHO/Michelson Doppler Imager (MDI) and SDO/Helioseismic and Magnetic Imager (HMI)), the $\mathrm{CH}$ polarity is determined and the $\mathrm{CHs}$ with positive and negative polarities are represented with red and blue color, respectively (Figures 3 and 4, third column panels). Note that the upper two wavelengths depict mostly a similar $\mathrm{CH}$ structure, both within the same instrument, and between EIT and AIA. On the contrary, the two lower wavelengths, especially the $171 \AA$ line, depict rather different $\mathrm{CH}$ structures. We also noticed that even for the same Carrington rotation, maps of some wavelengths (here

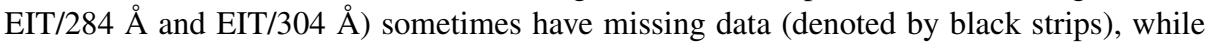
other wavelength maps are complete. This adds to the inconsistency between the $\mathrm{CH}$ maps and results based on the different wavelengths.

\section{Coronal Hole Dataset of McIntosh Archive}

Patrick McIntosh started creating hand-drawn synoptic maps in 1966. The first maps included various solar magnetic features based on daily $\mathrm{H} \alpha$ images. Using He I $10830 \AA$ images from the National Solar Observatory (NSO), CH boundaries were later (in 1973) added to the maps. Magnetograms were used, when available, to determine the dominant polarity of each region (Gibson et al., 2016). By compiling these maps, McIntosh developed a unique dataset showing the evolution of solar magnetic fields during Solar Cycles 20-23. Some versions of these maps were published as Upper Atmosphere Geophysics (UAG) reports and Solar-Geophysical Data (SGD) Bulletins. These reports were scanned and archived by NOAA National Center for Environmental Information (NCEI), and were recently used to produce the MacIntosh Archive (McA: https://www.ngdc.noaa.gov/stp/ space-weather/solar-data/solar-imagery/composites/synoptic-maps/mc-intosh/). The archive includes level-3 gif and fits files from CR1512 (11 September 1966) to CR2086 (23 July 2009). A large data gap is notable from CR1629 (08 June 1975) to CR1657 (10 July 1977).

Coronal hole observations exist from CR1601 (05 May 1973) to CR2086 (23 July 2009) with a total number of 486 synoptic maps (Table 2). Therefore, the McA dataset is overlapping only with the SOHO/EIT database. Figure 5 shows one of the McA original synoptic maps for CR1882 (29 April 1994). We extracted the CHs of the synoptic map and inverted the polarity colors to meet the convention used in SOHO/EIT and SDO/AIA maps (Figure 5, lower panel). The specific property of McA CH maps is that, although there is no direct polar observation, the polar $\mathrm{CHs}$ were manually extended to continuously cover the polar areas. Therefore, polar $\mathrm{CH}$ areas of McA maps do not show significant annual oscillation caused by limited solar visibility (the so-called vantage-point or b0-angle problem).

\section{Comparison of CH Datasets}

Coronal hole maps constructed from solar EUV images seriously suffer from the vantagepoint problem (e.g. Virtanen and Mursula, 2016; Hamada et al., 2018). As a result, the northern (southern) polar CHs have a poor visibility from Earth during spring (fall), when Earth is positioned at southern (northern) heliographic latitudes. As discussed above, the McA database, does not display the vantage-point effect because the polar regions are manually filled. However, the original He I observations, which the McA maps are based on, are 
A. Hamada et al.

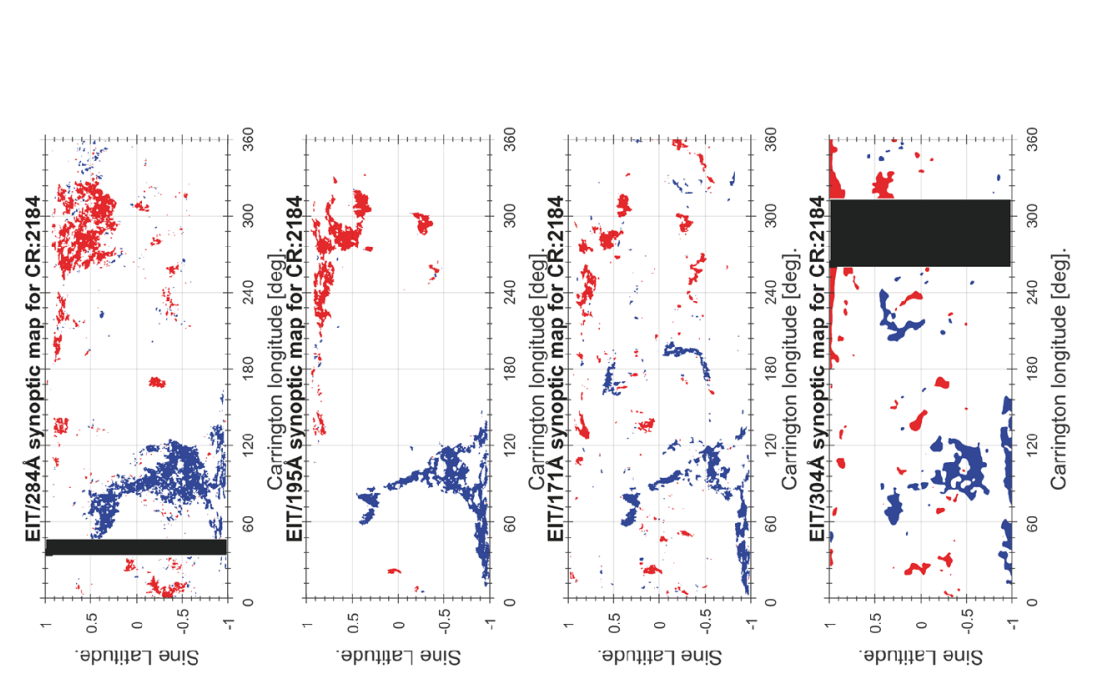

串串

$\varangle$

드모

产:

चี

退

$\circ$ ৫

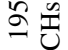

高亭

营哭

$\circ \stackrel{0}{2}$

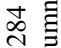

凷

핑 궁
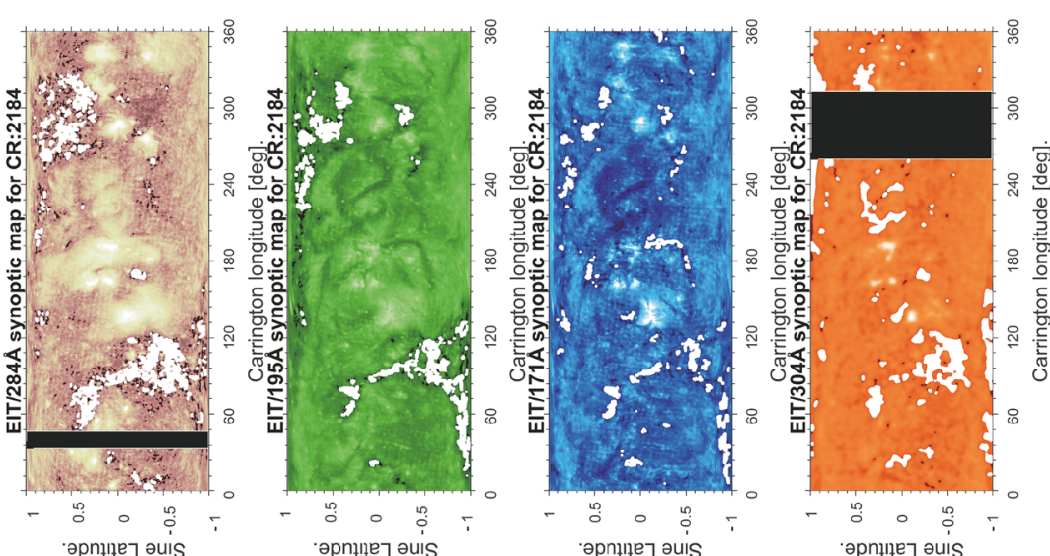

$\leftarrow$ I

再

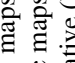

흔 융

ริ

की

隶

वे

월

要

芩焉

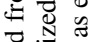

글

要

खे 웅

6
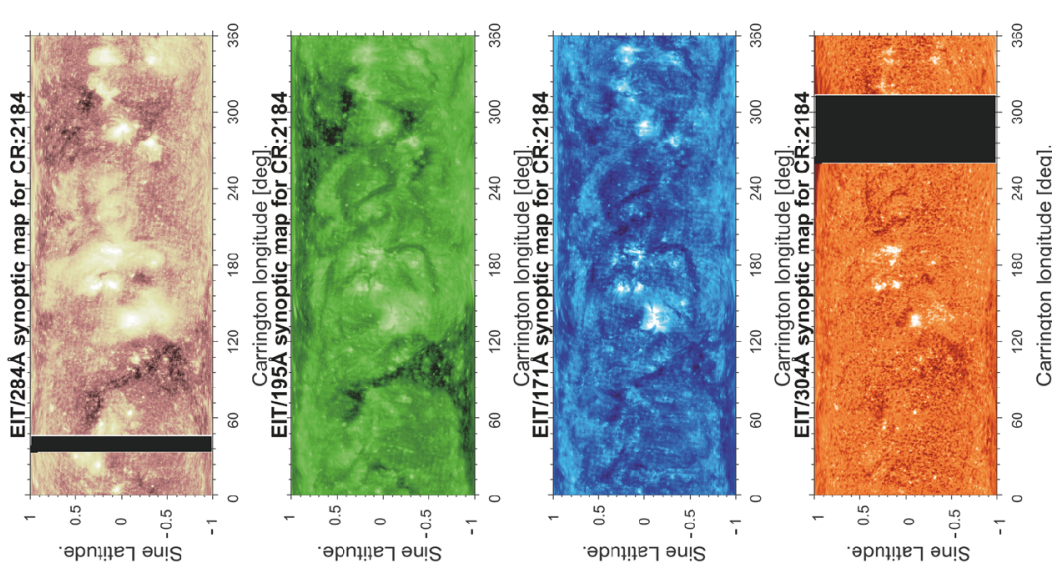

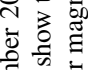

뭉

o

乙

믈

市 융

$\vec{\nabla}$.

웝

ㄴㅇㅇ

否字

प응 증

눌월

这吉造

다



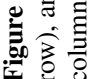



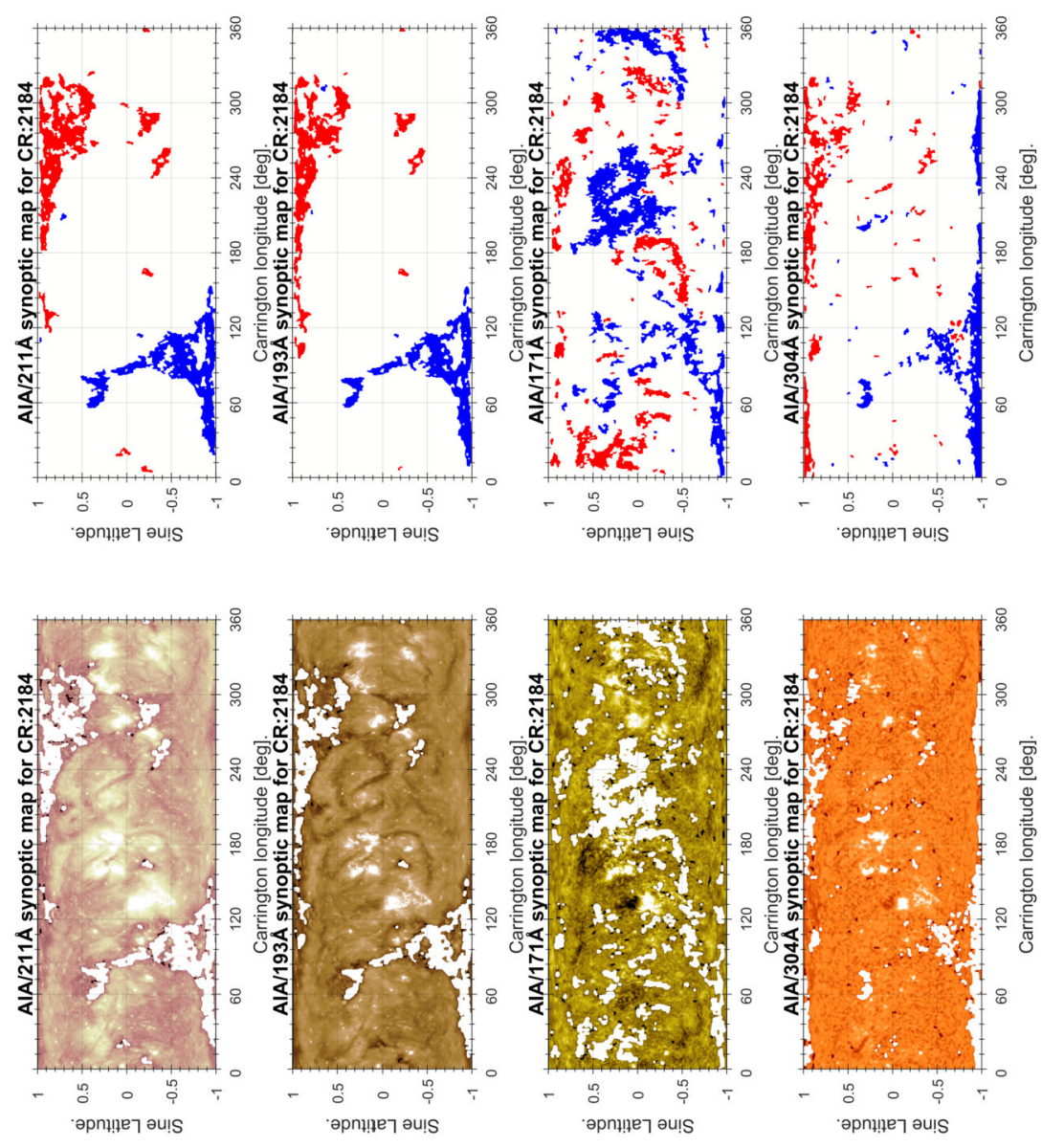


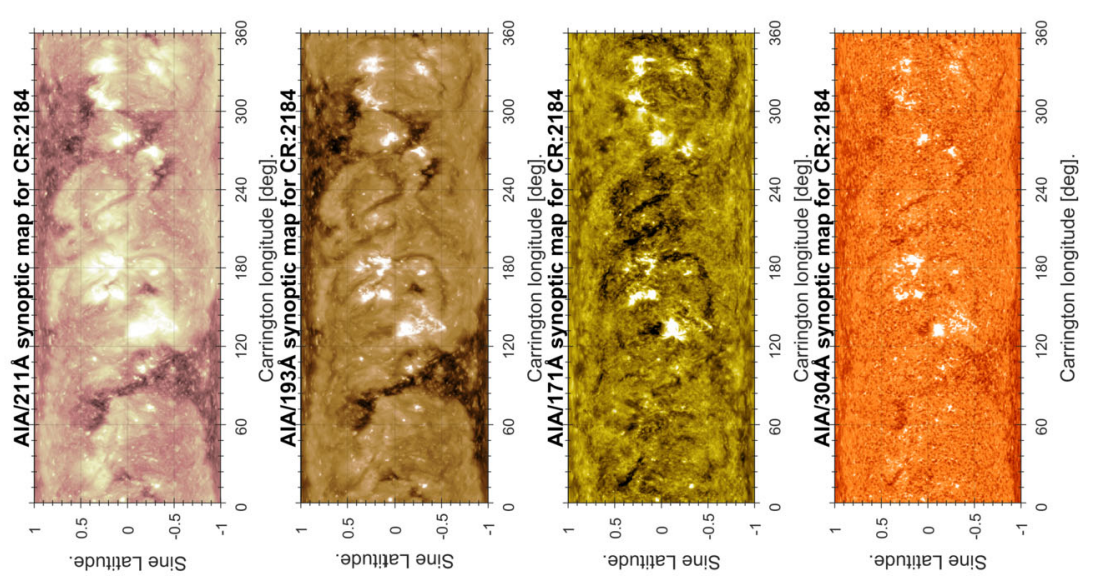

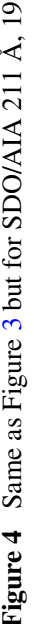



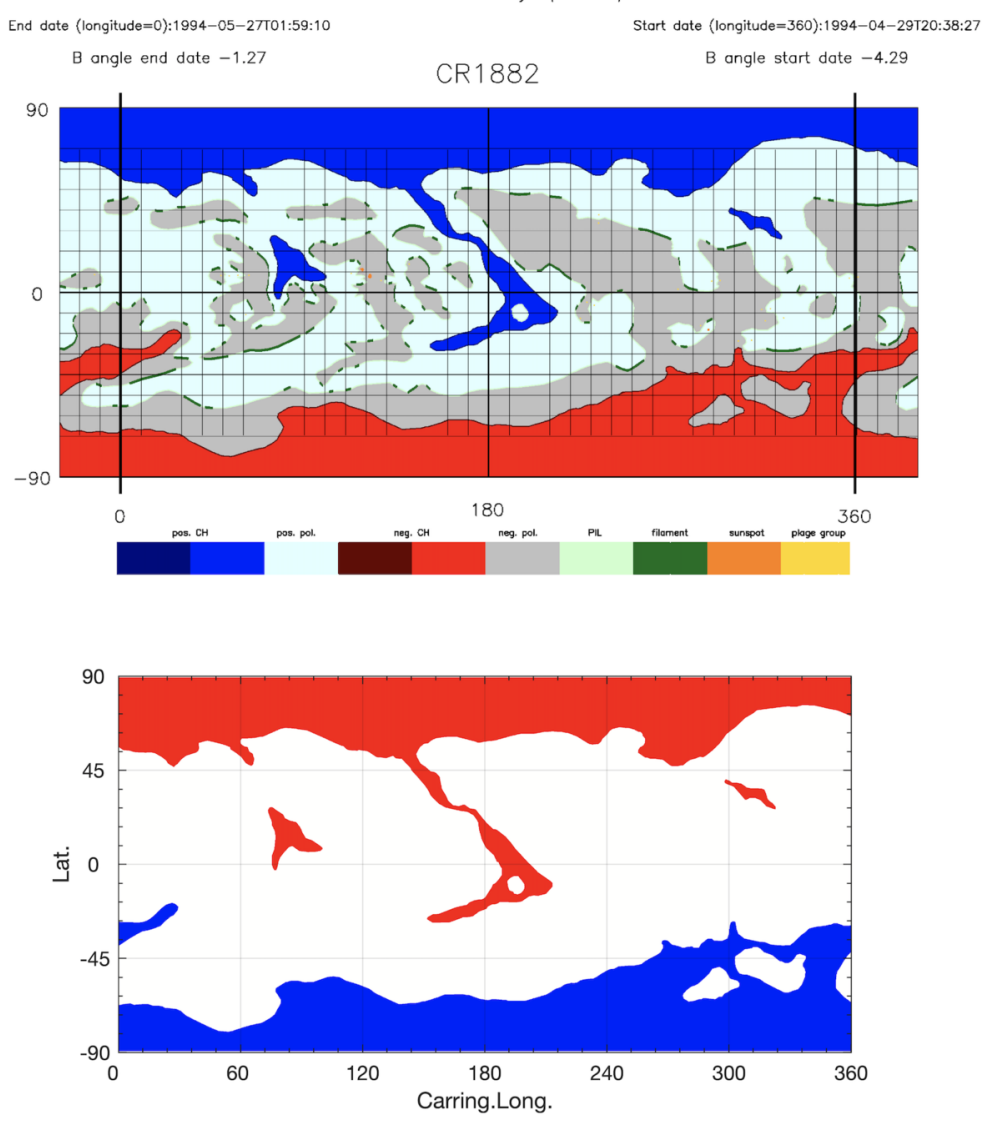

Figure 5 Original color coded McA synoptic map for CR1882 (29 April 1994) with negative (gray) and positive (light blue) polarity magnetic fields, negative (red) and positive (dark blue) CHs, and filaments (green). Extracted CH map with opposite color coding for magnetic polarities (red for positive and blue for negative).

also vulnerable to the effects of changing vantage points. Nevertheless, the vantage-point effect influences the EUV images and $\mathrm{CH}$ detection relatively more than the McA maps, because the effects of changing vantage point are not limited only to those polar latitudes which are completely out of sight in the less visible hemisphere but also extend to those high-latitude areas which are visible. At these latitudes the contrast between $\mathrm{CHs}$ and the surrounding regions is smaller than in the more visible pole; there is more coronal plasma obstructing the line-of-sight to the $\mathrm{CH}$ (see, e.g., Hess Webber et al., 2014). Our algorithm determines only one $\mathrm{CH}$ threshold intensity per map from the segment with the best contrast (which usually is either at low latitude or at the best visible pole). This leads to polar CHs not being detected so well on the less visible pole. Contrary to this, the $\mathrm{CHs}$ in McA data are traced by human observers, who can take into account the different contrasts at the opposing poles, and set the $\mathrm{CH}$ boundary at some contrast level and fill in the polar regions with unipolar field. Therefore, even though $\mathrm{McA} \mathrm{CH}$ maps have their uncertainties they serve as a good reference to which the EIT and AIA based $\mathrm{CH}$ maps can be compared.

The topmost color panel of Figure 6 shows the $\mathrm{McA} \mathrm{CH}$ areas at each latitude bin, normalized to the total solar surface. One can see that the (hand-filled) polar $\mathrm{CHs}$ (PCHs) dom- 


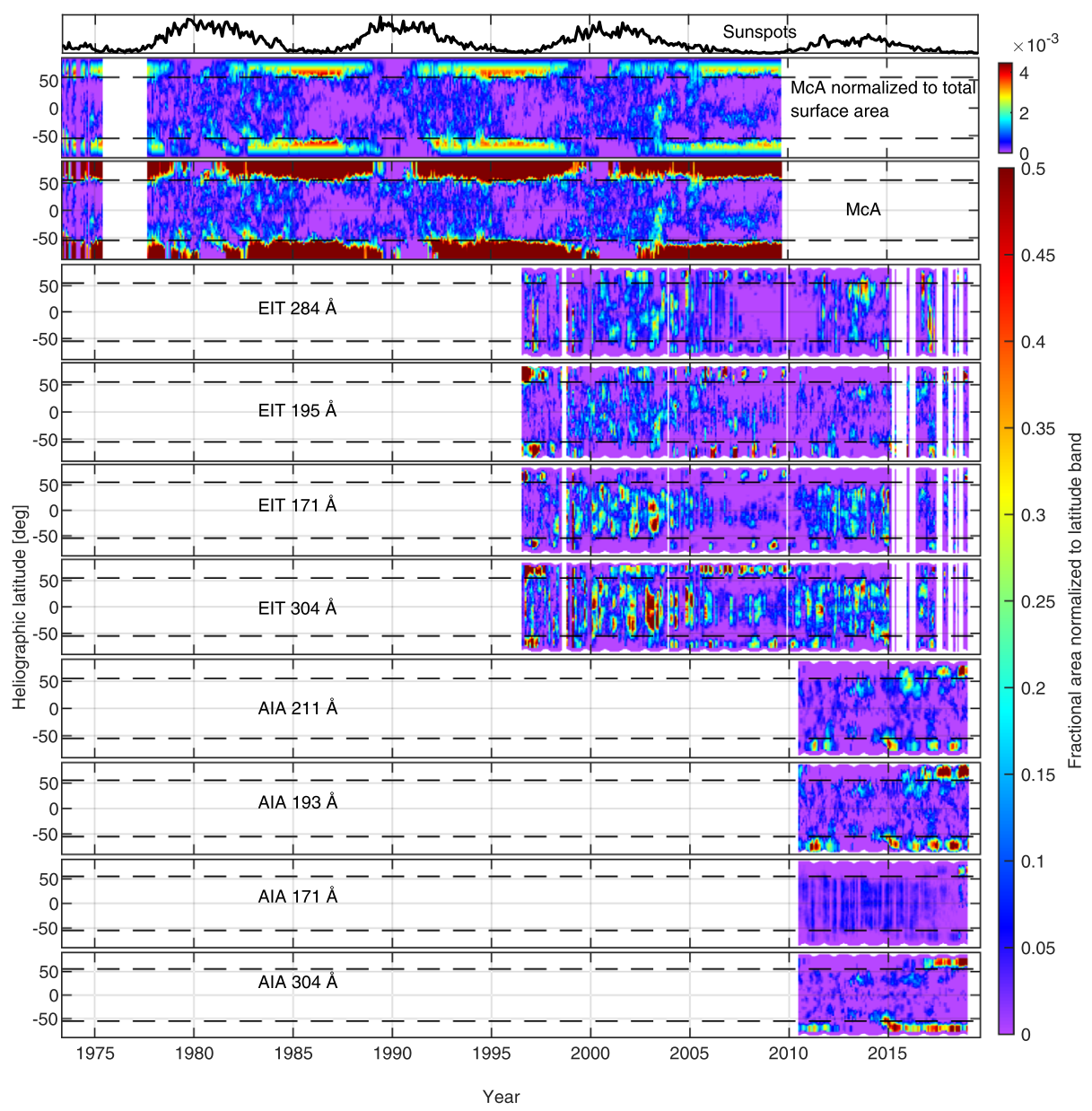

Figure $6 \mathrm{CH}$ areas at different latitudes through Solar Cycles 21, 22, 23, and 24. The top panel shows the monthly sunspot number for reference. The second panel from the top shows latitudinal McA areas normalized to the total solar surface. The color panels from the third panel down show fractional $\mathrm{CH}$ area normalized to the total area of the respective latitude band for McA, EIT $(284 \AA, 195 \AA, 171 \AA$, $304 \AA)$ and AIA $(211 \AA, 193 \AA, 171 \AA, 304 \AA)$, respectively. Horizontal black dashed lines at $\pm 55^{\circ}$ latitude distinguish polar and lower-latitude zones. Vertical white spaces denote missing data.

inate $\mathrm{CH}$ areas from the early descending phase to the ascending phase. PCHs are typically limited poleward from the $55^{\circ}$ latitude band (noted as a dashed line in Figure 6) where they also attain the largest (normalized) areas, while the PCH area shrinks toward the pole.

We compare the $\mathrm{CH}$ areas of EIT and AIA to the McA dataset and to each other with the aim of assessing which of the EIT and AIA wavelengths display CHs most systematically and show best agreement with McA thereby yielding a uniform long-term record of CHs. First the EIT and AIA CH maps are converted from sine latitude to linear latitude to match with McA dataset. For each dataset, the latitudinal distribution of fractional $\mathrm{CH}$ area is constructed. For each $\mathrm{CH}$ synoptic map, the fraction $\left(f_{C H}\right)$ of solar surface at each latitude 
bin $\lambda_{i}$ covered by $\mathrm{CHs}$ is calculated by

$$
f_{C H}\left(\lambda_{i}\right)=\frac{n_{i}}{N_{i}}
$$

where $n_{i}$ is the number of $\mathrm{CH}$ pixels at latitude $\lambda_{i}$ and $N_{i}$ is the total number of pixels at the same latitude.

The nine lower panels of Figure 6 show the latitudinal distribution of fractional $\mathrm{CH}$ area for all wavelengths of the three datasets. One can see in the second color panel of Figure 6 that the McA synoptic maps show the contiguous extent of $\mathrm{PCHs}$ from about $55^{\circ}$ latitude until exactly $90^{\circ}$ in both hemispheres during several years around solar minima. PCHs decline in the ascending phase and disappear for a couple of years around solar maxima. The EIT and AIA panels depict the seasonally varying extent of PCH observations, in anti-phase between the northern and southern hemispheres. Accordingly, EIT and AIA PCHs typically appear only during a few months around the favorable equinox. Moreover, they never fully cover the pole, contrary to McA, therefore increasing differences between the $\mathrm{PCH}$ observations.

Based on a visual inspection of Figure 6 the closest correspondence of polar CHs between McA and EIT is obtained for EIT/195 $\AA$ and $304 \AA$ wavelengths. This is seen both at the minimum of the late 1990s and during the whole declining phase of Solar Cycle 23. PCHs appear more systematically and are considerably larger in $195 \AA$ and $304 \AA$ than in the two other wavelengths $211 \AA$ and $171 \AA$. Note also that McA PCHs appear earlier (in 2001) in the north than in the south (in 2003), which is followed by the EIT PCHs, especially in the two best wavelengths $195 \AA$ and $304 \AA$.

AIA shows the PCHs fairly similarly in three wavelengths (211 $\mathrm{A}, 193 \AA$, and $304 \AA$ ). Only the $171 \AA$ line finds almost no PCHs. As for EIT, the $193 \AA$ and $304 \AA$ lines depict the PCHs most systematically. All the three wavelengths show an earlier start of $\mathrm{PCH}$ evolution in the south during Solar Cycle 24, and even the latitudinal evolution of the large $\mathrm{CH}$ moving from the mid-latitudes to the south pole in 2014-2015. Overall, AIA shows the PCHs more clearly and systematically than EIT, not only due to the data gaps at the end of EIT time interval. AIA $211 \AA$ is superior to EIT $284 \AA$ in PCH detection, but AIA is better in PCH detection than EIT even in the two best lines (193/195 $\AA$ and $304 \AA$ ).

The low-latitude $\mathrm{CH}(\mathrm{LLCH})$ areas peak during the solar maximum and early declining phase, and have a minimum around solar minima. It can be seen on the McA plots of Figure 6 that LLCHs almost disappear at the end of Solar Cycles 21 and 22. However, the LLCHs remain fairly sizable at the minimum between Solar Cycles 23 and 24. The long persistence of LLCH during Solar Cycle 23 is one of the peculiar features of this cycle (Gibson et al., 2016). At low latitudes the EIT $171 \AA$ and $304 \AA$ wavelengths show excessive and likely erroneous low-latitude $\mathrm{CH}$ areas compared to McA during most of the time interval. The $284 \AA$ wavelength gives a much closer agreement with McA LLCH areas except for the late descending phase of Solar Cycle 23 and the subsequent minimum, where it fails to detect almost any LLCHs. The $195 \AA$ wavelength shows closest agreement with McA also for LLCHs, with the absolute LLCH level, as well as its spatial-temporal evolution being very similar to McA LLCH areas throughout the whole interval, including the minimum between Solar Cycles 23 and 24.

The two AIA lines, $211 \AA$ and $193 \AA$, display mutually a closely similar LLCH evolution, which also agrees well with the EIT $195 \AA$ LLCH observations. However, the AIA $211 \AA$ and EIT $284 \AA$ LLCH observations are quite different. The two other wavelengths, $171 \AA$ and $304 \AA$, also depict surprisingly different LLCH results for EIT and AIA, despite being the same wavelengths. Apparently, the contrast of different solar features even in the same 

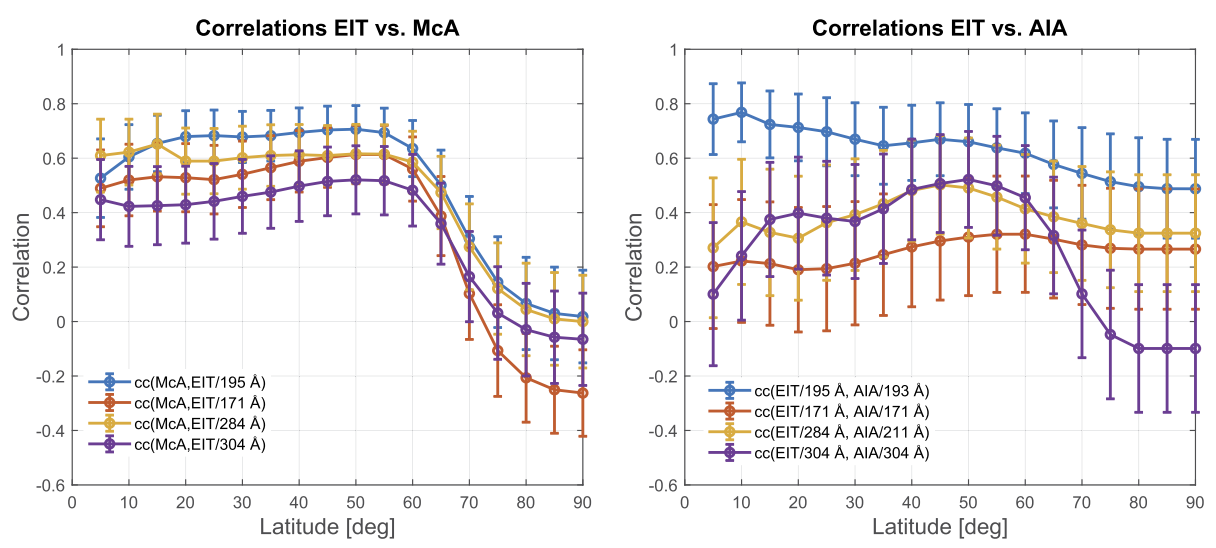

Figure 7 Left: Correlations between McA and EIT fractional $\mathrm{CH}$ areas computed for a symmetric latitude band around the equator up to the latitude depicted on the X-axis. Right: The same between EIT and AIA. The different colors indicate different wavelengths. The error bars depict the $95 \%$ confidence limits of the correlations.

wavelength is different in the two instruments, most likely due to instrumental reasons, e.g., differences in image contrast which are not compensated by the overall intensity histogram transformations employed by Hamada, Asikainen, and Mursula (2019). The AIA $193 \AA$ Aine and EIT $195 \AA$ give the closest agreement even for LLCH, when the four corresponding EIT/AIA wavelengths are compared. Accordingly, this preliminary comparison suggests that the AIA $193 \AA$ line gives the most reliable estimate of LLCH areas during Solar Cycle 24, and could be used to continue the McA/EIT $195 \AA$ ALCH areas.

\section{Latitudinal Correlation of LLCH Areas}

In order to quantify how well the EIT and AIA LLCH areas correspond to each other or to the McA dataset, we computed the correlation coefficients of fractional $\mathrm{CH}$ areas from the equator to several different latitudes (symmetrically in both hemispheres) between the two datasets. These correlations are shown in Figure 7 as a function of the limiting latitude. The error bars indicate the $95 \%$ confidence limits for the correlation coefficients. The correlations have been computed only for those synoptic maps, which do not have data gaps (apart from those at the polar regions due to the vantage-point effect). The left side plot of Figure 7 shows the correlations between the four EIT wavelengths and the McA dataset. One can see a systematic pattern for all wavelengths so that the correlations are fairly constant (or slightly increase) up to $55^{\circ}$ latitude beyond which they quickly drop close to zero. This drop in correlation is obviously caused by the very different treatment of PCHs in the two datasets discussed already above. The plot therefore indicates that the vantage-point effect begins to affect the observability of $\mathrm{CHs}$ above $55^{\circ}$ heliographic latitude. The left side plot of Figure 7 also indicates that the $195 \AA$ wavelength correlates best with the McA dataset over most latitude bands, while the $304 \AA$ line is least successful at low latitudes and the $171 \AA$ A line fails worst at high latitudes.

The right side plot of Figure 7 shows the correlations between the fractional $\mathrm{CH}$ areas based on corresponding EIT and AIA wavelengths. It should be noted that the correlation between EIT and AIA are based on a much shorter time series than the correlations between 
Figure 8 Correlations between McA and EIT $195 \AA$ (blue) and EIT $195 \AA$ and AIA $193 \AA$ (red) fractional $\mathrm{CH}$ areas computed separately for $5^{\circ}$ latitude bands around the latitude depicted on the $\mathrm{X}$-axis.

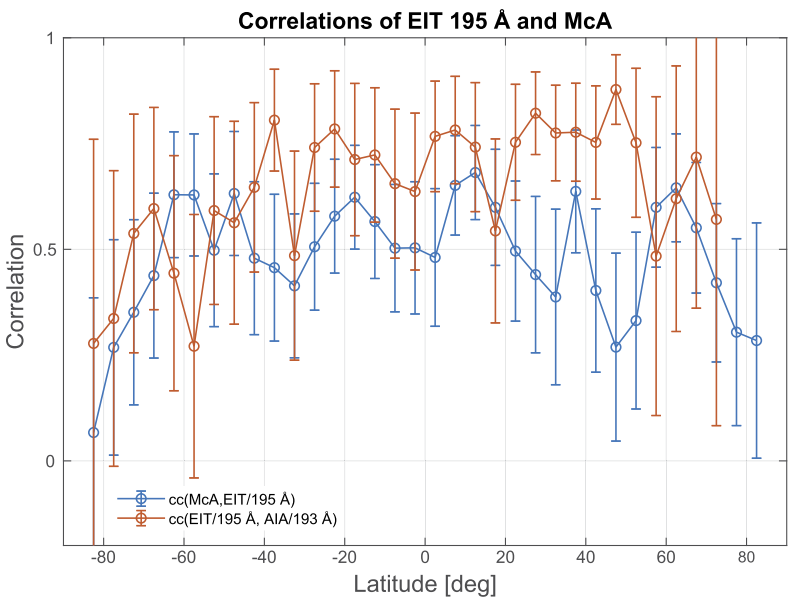

EIT and McA due to significant data gaps in EIT after 2015 (see Figure 6). Figure 7 shows a good correlation ( $\mathrm{cc} \geq 0.5$ ) between EIT/195 $\AA$ and AIA/193 $\AA$ for all latitude limits, with a slight decrease in correlation towards the poles. All other wavelengths display smaller correlations. As already seen in Figure 6, there is a poor correlation between EIT/AIA $171 \AA$ wavelengths even at low latitudes. In fact, this correlation remains insignificant for almost all latitude limits. For EIT/284 $\AA$ and $\mathrm{AIA} / 211 \AA$ the correlation is fair $(\mathrm{cc} \approx 0.4)$ for all latitude limits. For EIT/AIA $304 \AA$ the correlations are fair $(\mathrm{cc} \approx 0.4)$ until $60^{\circ}$ latitude, but drop to zero poleward of this latitude limit. This verifies the above discussion that the PCHs are depicted very differently by EIT and AIA in this wavelength. It should also be noted that because of the many data gaps of EIT since 2015, the simultaneous measurements of EIT and AIA are effectively limited to the ascending and maximum phase of Solar Cycle 24 when the $\mathrm{CHs}$ have a small area. This naturally also increases the influence of random errors on the correlations. Overall, Figure 7 confirms that the most systematic correlations of CHs at all latitudes are found for the EIT/195 $\AA$ and AIA/193 $\AA$ wavelengths.

Figure 8 shows the correlations between EIT $195 \AA$ and McA and EIT $195 \AA$ and AIA $193 \AA$ calculated separately for each $5^{\circ}$ wide latitude band. For EIT $195 \AA$ and McA, correlations remain fairly high (except for some latitude bins) until about $60^{\circ}$ latitude in both hemispheres, whereafter they drop to low values. For EIT $195 \AA$ and AIA $193 \AA$ the correlations are very good until $50^{\circ}$ in north and $40^{\circ}$ south, beyond which they drop to the same level as $195 \AA$ vs. McA correlations. We note that the overall correlation of LLCH areas extracted automatically from EIT $195 \AA$ and AIA $193 \AA$ is higher that the corresponding correlation between the manually-based McA and the automated EIT $195 \AA$.

\section{Joining the $\mathrm{CH}$ Series}

Figure 8 demonstrates that low-latitude CHs (based on 195/193 A lines) do not suffer significantly from the vantage-point effect up to about $\pm 55^{\circ}$ latitude. Therefore we computed the total fractional LLCH areas between $\pm 55^{\circ}$ latitude, and separately the two $\mathrm{PCH}$ areas above $55^{\circ}$ in both hemispheres (north $\mathrm{PCH}$ and south $\mathrm{PCH}$ ). Figure 9 depicts these three time series for these three datasets (along with the sunspot number for reference). One can see that in all three datasets, the polar CHs maximize during the late declining to minimum 
(a)



(b)

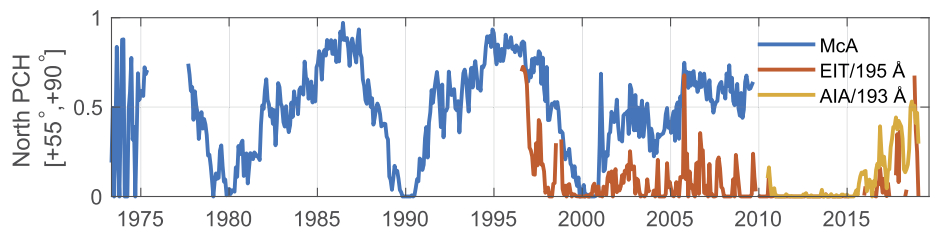

(c)



(d)

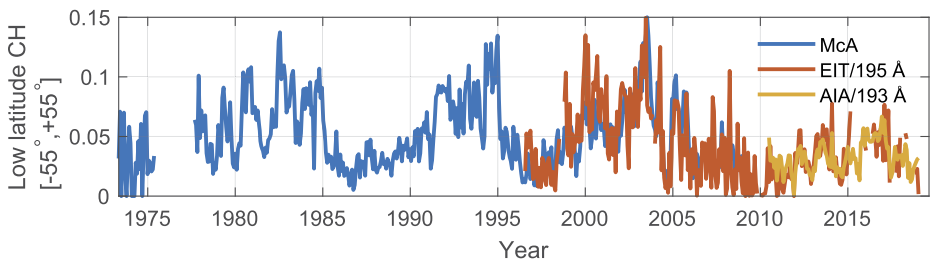

Figure 9 Time series of fractional areas of CHs compared with monthly sunspot numbers in the top panel. Northern (a) and southern (b) polar $\mathrm{CH}(\mathrm{PCH})$ areas between latitudes $\pm 55^{\circ}$ and $\pm 90^{\circ}$, from McIntosh (blue), EIT/195 ̊̊ (red) and AIA/193 ^̊ (yellow) dataset. (c) Low-latitude CH (LLCH) areas between latitudes $+55^{\circ}$ and $-55^{\circ}$.

phase of the solar cycle, and practically vanish during the solar maximum, in accordance with the well known evolution of the solar magnetic cycle.

As discussed above, the PCHs observed, e.g., by EIT $195 \AA$ A yield a reasonable estimate of $\mathrm{PCH}$ areas only during the short favorable season of good visibility of the respective pole (north in fall, south in spring). Accordingly, the total (or annual) PCH areas from EIT do not match well with the hand-filled all-year PCH estimates of McA series. However, we note that the EIT PCH seasonal maxima often reach the same level as the McA PCH for the same time. This is true, e.g., for the EIT north PCH peaks in 1996 and 2006 and south PCH peaks in 1997 and 2008. We also note that the time evolution of the EIT PCH seasonal peaks follows quite well the time evolution of the corresponding McA PCH curve. This is particularly clear in the north from the start of EIT PCH in 1996 until 2007, and in the south from 1996 to 2000. Only in the north the decrease of EIT peaks from 2006 to 2009 is not seen in McA PCH. Accordingly, despite the fact that the EIT PCH peaks do not always reach the same level as $\mathrm{McA} \mathrm{PCH}$ areas, they mostly agree with the long-term evolution of the McA PCH.

Figure 9 shows that the PCH areas from EIT $195 \AA$ and AIA $193 \AA$ follow each other quite well. We have studied this in more detail in Figure 10, which shows a scatter plot between annual peaks of fractional EIT PCH areas (separately for northern and southern 
$\mathrm{PCH}$ ) and simultaneous McA PCH areas. The values for the northern hemisphere are shown in blue and for the southern hemisphere in red. The EIT PCH peaks were identified from the time series of Figure 9 by first identifying all local peaks and then selecting those, which are largest within 1-year window around the corresponding peak. One can see that both hemispheres follow the same overall relationship, which is roughly linear at large values of the fractional area, but at small values the relationship shows clear non-linearity. The overall relationship was found to be well approximated by a semi power-law fit, which is indicated for illustrational purposes in the plot by the green curve. Using the non-linear Spearman rank method (Zar, 2014), one finds that the correlation between the EIT peaks and McA values is highly significant $\left(\mathrm{cc}=0.87, \mathrm{p}=10^{-6}\right)$. In Figure 10 one can also see an apparent minimum value of EIT PCH fractional area of 0.067 . This most likely reflects the observational threshold $\mathrm{CH}$ size in the EIT synoptic maps. The algorithm by Hamada et al. (2018) filters out very small CHs.

Figure 9 shows that there is a remarkably good correspondence in low-latitude $\left( \pm 55^{\circ}\right)$ $\mathrm{CH}$ areas between McA, EIT, and AIA. This is further demonstrated in Figure 11 displaying the scatter plots of fractional LLCH areas (for a slightly larger bin between $\pm 60^{\circ}$ ) between McA and EIT and AIA and EIT. In both cases the correlations are good and highly significant $\left(\mathrm{cc}=0.64, \mathrm{p}=7.8 \times 10^{-17}\right.$ for McA/EIT and $\mathrm{cc}=0.62, \mathrm{p}=1.2 \times 10^{-8}$ for AIA/EIT). In Figure 9 all three datasets show that low-latitude $\mathrm{CHs}$ start to grow from a sunspot minimum towards a maximum in the mid-declining phase. Comparing the three datasets one can clearly see that, in addition to the good correlation evident in Figure 8, the overall levels of low-latitude CHs in Figure 9 are very similar in EIT $195 \AA$, AIA $193 \AA$, and McA. This indicates that McA, EIT $195 \AA$, and AIA $193 \AA$ can be used to form a uniform series of low-latitude CHs.

It is interesting to find in Figure 9 that the peaks of the low-latitude $\mathrm{CHs}$ in the declining phase of Solar Cycles 21, 22, and 23 are all roughly at the same level, but the latest Solar Cycle 24 displays a considerably (about a factor of 2) smaller maximum of low-latitude $\mathrm{CHs}$ than these previous cycles. The maximum size of low-latitude $\mathrm{CHs}$ therefore seems to follow the amplitude of the sunspot cycle. This suggests that the amount of LLCHs is related to the number of sunspot-related active regions, emerging during the active region decay by diffusion and reconnection.

The PCH and LLCH time series discussed above are fairly consistent with those presented by Lowder et al. (2014) who studied PCH and LLCH areas extracted from full-disk EUV images of SOHO/EIT (1996 to 2011) and from combined observations of SDO/AIA and STEREO/EUVI A/B (May 2010 to January 2013). They also used segmented image thresholding technique to find the $\mathrm{CH}$ boundaries. Additionally, they found a notable vantage-point effect in the extracted $\mathrm{PCH}$ areas. PCHs were also studied by Hess Webber et al. (2014) using manually assisted thresholding of EUV synoptic maps, specifically tuned to extract polar CHs. They also found the vantage-point effect to influence $\mathrm{PCH}$ areas, but their $\mathrm{PCH}$ areas did not drop entirely to zero during the disfavorable seasons. Their annually averaged $\mathrm{PCH}$ areas showed the same solar cycle evolution of polar $\mathrm{CHs}$, which is seen also in the McA PCH areas here. Hess Webber et al. (2014) also found that PCHs extracted from $304 \AA$ seem to be least vulnerable to the vantage-point effect. It is likely that our and Lowder et al. (2014) results for PCH areas differ from those of Hess Webber et al. (2014) because of the differences in determining the $\mathrm{CH}$ threshold intensity. Hess Webber et al. (2014) specifically concentrated on polar areas, while our algorithm considers the entire solar surface when searching for the optimal image segment to extract the $\mathrm{CH}$ threshold intensity. Threshold intensities extracted from $\mathrm{CHs}$ at lower latitudes, where the contrast is typically better, may lead into underestimating $\mathrm{PCH}$ areas in the less visible hemisphere, 
Figure 10 Correlation between the peak EIT $195 \AA$ PCH fractional areas and simultaneous McA LLCHs. Blue points depict the northern hemisphere and red points the southern hemisphere. Green line presents the best fit curve to these points.



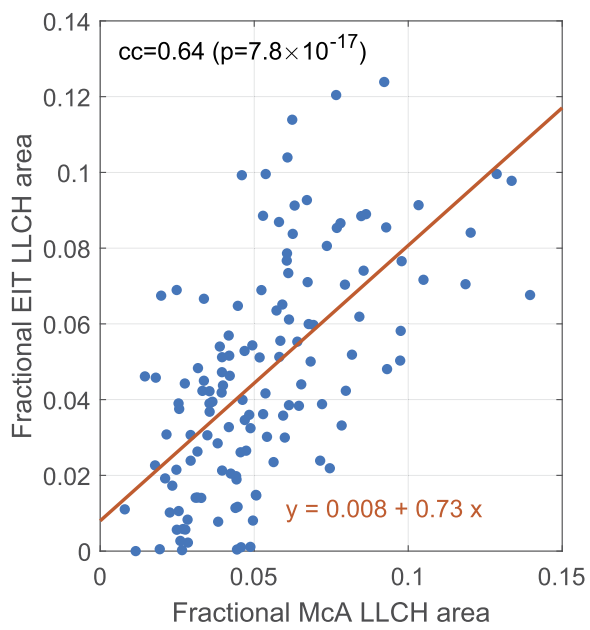

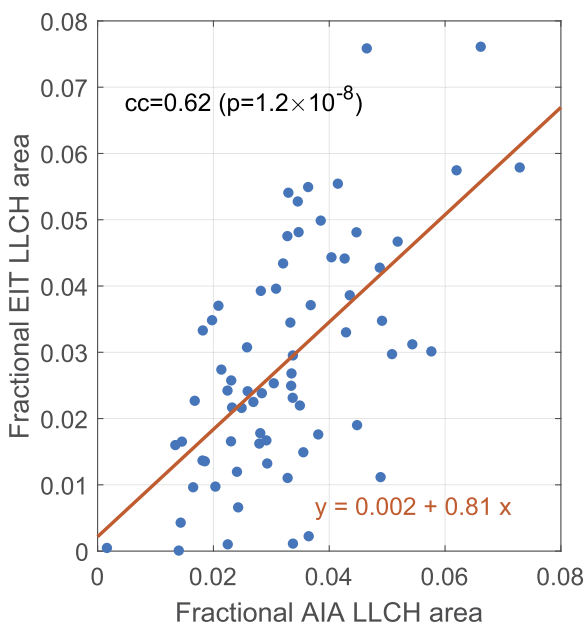

Figure 11 Left: Correlations between $\mathrm{McA}$ and EIT fractional $\mathrm{CH}$ areas computed for the latitude band between $\pm 60^{\circ}$ (low-latitude CHs). Right: The same between EIT and AIA.

where the contrast is smaller. These comparisons indicate that the best approach might be to use a different $\mathrm{CH}$ threshold for polar and lower latitudes, or to pre-process the synoptic maps by some form of local histogram equalization. However, these improvements are outside the context of the present study.

Based on the above analyses it is clear that we can obtain a fairly uniform long-term record of LLCHs using McA, EIT $195 \AA$, and AIA $193 \AA$ wavelengths. Therefore, we constructed a composite of McA, EIT $195 \AA$, and AIA $193 \AA$ in eight different $15^{\circ}$-wide latitude bands between $\pm 60^{\circ}$ as well as for the entire $\pm 60^{\circ}$ latitude band. Even though the three datasets display good correlation with each other at these latitudes they need to be linearly scaled to the same level before joining them into a composite record. We scaled both McA 
and AIA $193 \AA$ fractional LLCH area time series separately in each latitude band to the corresponding EIT $195 \AA$ level. This was done by fitting a line $f_{E I T}=a_{A I A}+b_{A I A} \times f_{A I A}$ between EIT and AIA using those simultaneous points, where the synoptic maps do not have any data gaps. This equation was then used for all AIA values to scale them to EIT level. Then a similar fit was done between McA and EIT, i.e. $f_{E I T}=a_{M c A}+b_{M c A} \times f_{M c A}$. Table 3 shows the scaling parameter values used to convert AIA and McA LLCH areas to EIT level along with their estimated standard deviations. These scaling parameters were then applied to the entire McA and AIA LLCH time series of the corresponding latitude band. It should be noted that the scaling parameters are not generally usable but apply only with the specific algorithm and EUV processing discussed in this article. One can see that the $b_{M C A}$ scaling factors at the highest latitude bins are smaller than at lower-latitude bins. A possible reason for this is that no correction to center-to-limb variation in brightness was employed in the full-disk images used to construct the EUV synoptic maps. Such limb-brightening corrections were performed, e.g., by Schrijver and McMullen (2000), Suresh (2013), Verbeeck et al. (2014), Caplan, Downs, and Linker (2016). Accounting for the center-to-limb variation would likely enhance contrast at high latitudes and lead to larger $\mathrm{CH}$ areas and to larger $b_{M c A}$ factors at high latitudes. The similar latitudinal tendency of $b_{A I A}$ factors is likely also caused by differences in image contrast between EIT and AIA instruments, which are accentuated towards the pole by different center-to-limb variations.

The composite data series in each latitude band was then formed from the scaled McA time series until 23 July 2009, the EIT time series between 23 July 2009-20 May 2010 and the scaled AIA time series after 20 May 2010. Figure 12 displays these combined LLCH time series for the eight different latitude bands (panels with blue curves) and for the entire $\pm 60^{\circ}$ latitude band (middle panel with red curve). For comparison the central panel also displays the unscaled McA (green curve) and unscaled AIA (violet curve) fractional areas. It should be noted here that each plot shows the fraction of total area of the corresponding latitude band covered by $\mathrm{CHs}$, and that the total areas of the different latitude bands are not equal. The composite LLCH series of Figure 12 shows best the overall evolution of lowlatitude CHs, which peaks in the declining phase of the solar cycles in 1982, 1994, 2003, and 2016. It also shows that these peaks of fractional LLCH areas are roughly equally high in Solar Sycles 21-23. However, the maximum LLCH area of Solar Cycle 24 is roughly half of those of the previous ones, as already seen in the unscaled time series in Figure 9. One can also see from the central panel that the scaling hardly changes the AIA fractional areas, which means that LLCHs extracted from AIA observations in the future will be directly comparable to the composite presented here.

\section{Summary and Conclusion}

In this study we presented new long-term $\mathrm{CH}$ distributions based on the recently constructed homogenized SOHO/EIT (1996 to 2018) and SDO/AIA (2010 to 2018) synoptic EUV maps, and the McIntosh Archive CH dataset (1973-2009). The CHs from EIT and AIA maps were identified by the automated algorithm developed by Hamada et al. (2018). Comparing the CHs extracted from four EIT wavelengths ( $284 \AA, 195 \AA, 171 \AA$ and $304 \AA$ ) we found that the EIT $304 \AA$ and $195 \AA$ lines give the best estimate of polar CHs (within the limitation set by the vantage-point effect), while the two other wavelengths $284 \AA$ and, especially, $171 \AA$ have difficulties in revealing polar CHs. On the other hand, the EIT $284 \AA$ and $195 \AA$ lines give the best estimate of low-latitude $\mathrm{CHs}$ between $-60^{\circ}$ and $+60^{\circ}$. Accordingly, we found 


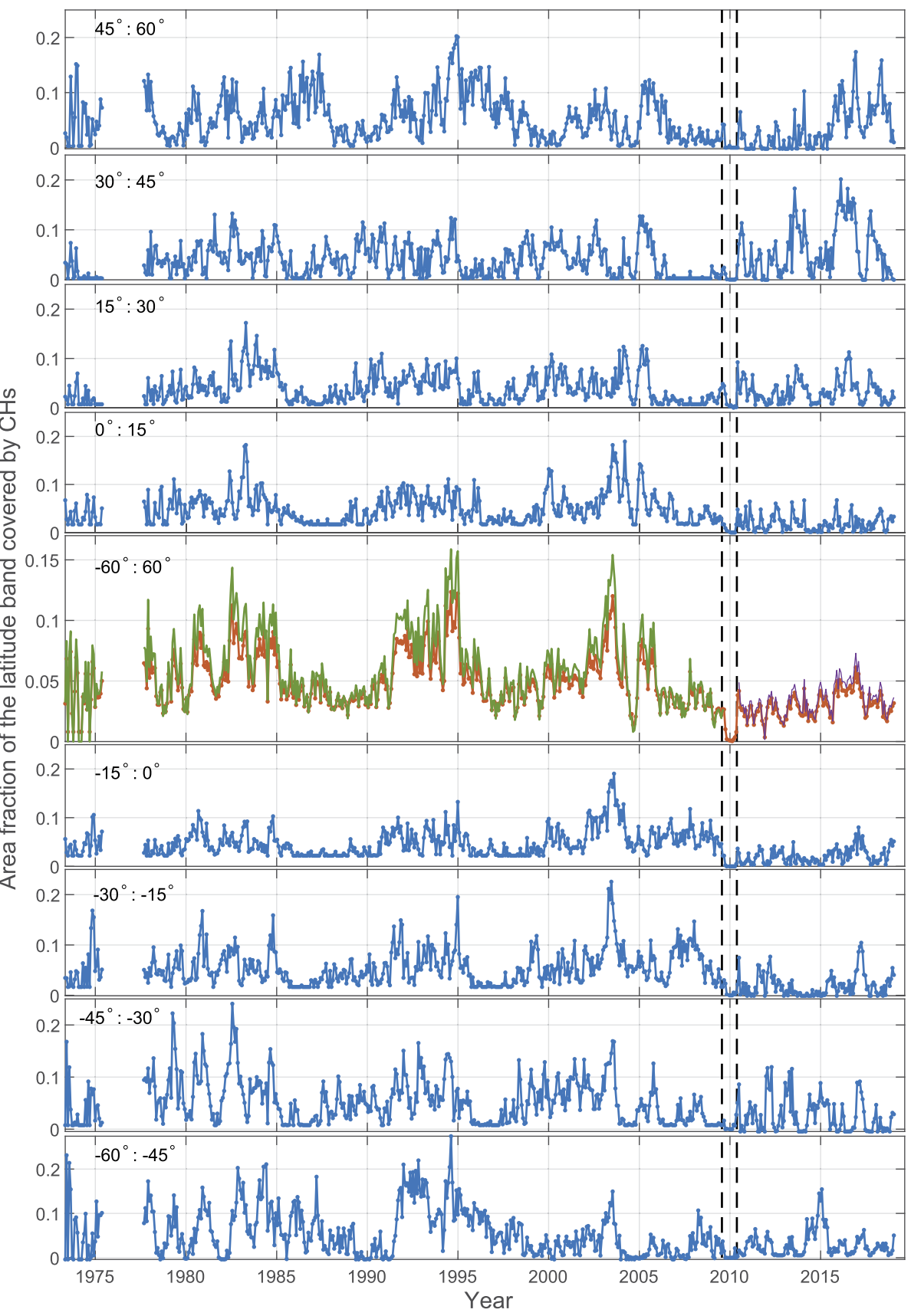

Figure 12 Composite time series of fractional $\mathrm{CH}$ area separately in $15^{\circ}$ latitude bands ranging from $-60^{\circ}$ to $60^{\circ}$ heliographic latitude. The central panel with the red curve displays the fractional $\mathrm{CH}$ area of the entire $\pm 60^{\circ}$ latitude band. The composite has been constructed from McA data up to 23 July 2009 (left vertical dashed line), EIT data between 23 July 2009 - 20 May 2010 (right vertical dashed line), and AIA data after 20 May 2010. McA and AIA fractional areas in each latitude band have been scaled to EIT level. For comparison the central panel also displays the unscaled McA (green curve) and unscaled AIA (violet curve) fractional areas. 
Table 3 Scaling factors to convert AIA and McA to EIT level. Each column shows the parameter values and their standard deviations.

\begin{tabular}{lrlrl}
\hline Latitude band & \multicolumn{1}{l}{$a_{A I A}$} & \multicolumn{1}{l}{$b_{A I A}$} & \multicolumn{1}{l}{$a_{M c A}$} & \multicolumn{1}{l}{$b_{M c A}$} \\
\hline $45^{\circ}: 60^{\circ}$ & $-0.0020 \pm 0.0043$ & $0.6937 \pm 0.0626$ & $0.0031 \pm 0.0073$ & $0.39 \pm 0.058$ \\
$30^{\circ}: 45^{\circ}$ & $-0.0003 \pm 0.0053$ & $0.9909 \pm 0.0772$ & $0.0026 \pm 0.0046$ & $0.73 \pm 0.076$ \\
$15^{\circ}: 30^{\circ}$ & $0.0059 \pm 0.0032$ & $0.8539 \pm 0.0793$ & $0.0070 \pm 0.0041$ & $0.86 \pm 0.086$ \\
$0^{\circ}: 15^{\circ}$ & $0.0011 \pm 0.0021$ & $0.9000 \pm 0.0761$ & $0.0170 \pm 0.0044$ & $0.85 \pm 0.077$ \\
$-60^{\circ}: 60^{\circ}$ & $0.0022 \pm 0.0044$ & $0.8097 \pm 0.1250$ & $0.0080 \pm 0.0046$ & $0.73 \pm 0.076$ \\
$-15^{\circ}: 0^{\circ}$ & $0.0036 \pm 0.0029$ & $0.7602 \pm 0.0856$ & $0.0220 \pm 0.0057$ & $0.71 \pm 0.080$ \\
$-30^{\circ}:-15^{\circ}$ & $-0.0010 \pm 0.0028$ & $1.0873 \pm 0.0887$ & $0.0170 \pm 0.0063$ & $0.82 \pm 0.086$ \\
$-45^{\circ}:-30^{\circ}$ & $-0.0045 \pm 0.0046$ & $1.0601 \pm 0.0959$ & $0.0077 \pm 0.0057$ & $0.77 \pm 0.077$ \\
$-60^{\circ}:-45^{\circ}$ & $0.0054 \pm 0.0047$ & $0.4679 \pm 0.0580$ & $-0.0039 \pm 0.0052$ & $0.51 \pm 0.056$ \\
\hline
\end{tabular}

that the EIT $195 \AA$ line shows the closest correspondence with the McA data on both polar and low-latitude CHs.

Comparing the four EIT lines with the corresponding AIA lines $(211 \AA, 193 \AA, 171 \AA$ and $304 \AA$ ) we found surprisingly large differences. The AIA $171 \AA$ line detected hardly any $\mathrm{CHs}$, whether polar or low-latitude, even much less than the EIT $171 \AA$ line. The AIA $304 \AA$ line detected polar CHs much better than EIT $304 \AA$ line, but produced a slightly too small amount of low-latitude CHs. The AIA $211 \AA$ and $193 \AA$ lines give a very similar view of both polar $\mathrm{CHs}$ and low-latitude $\mathrm{CHs}$. Both of these two lines best agree on $\mathrm{CHs}$ of EIT $195 \AA$ line, even AIA $211 \AA$ which measures roughly the same emission region as EIT $284 \AA$ line. Accordingly, the $195 \AA / 193 \AA$ lines give the most credible extension of McA $\mathrm{CH}$ series.

We compared the EUV based $\mathrm{CH}$ maps with the long-running McIntosh Archive $\mathrm{CH}$ dataset (1973 to 2009), which is based on CHs manually identified from He I emission line. The aim was to assess which EIT/AIA wavelengths depict CHs most systematically and show the best agreement with the McIntosh Archive thereby, with suitable scaling, yielding a uniform series of $\mathrm{CHs}$ from 1979 to 2018 . We found that the correlation between the fractional $\mathrm{CH}$ areas of all four EIT and AIA wavelengths with those of McA dataset begin to decrease rapidly when the considered latitude band is extended poleward of $60^{\circ}$ in both hemispheres. This indicates that, because of the vantage-point effect, the EIT and AIA EUV observations are not straightforwardly suitable for continuous polar $\mathrm{CH}$ monitoring. Out of the four wavelengths we found EIT $195 \AA$ and AIA $193 \AA$ to correlate best with McA when considering low-latitude CHs. Using the best-fitting EIT $195 \AA$ and AIA $193 \AA$ A based low-latitude $\mathrm{CH}$ series, we constructed a combined low-latitude $\mathrm{CH}$ series by scaling $\mathrm{McA}$ and AIA $\mathrm{CH}$ fractional areas to the corresponding EIT series. This scaling was performed separately at eight different $15^{\circ}$-wide latitude bands from $-60^{\circ}$ to $+60^{\circ}$, as well as for the whole $\pm 60^{\circ}$ band as one full low-latitude CH series. McA, EIT $195 \AA$, and AIA $193 \AA$ observations then form a uniform long-term series of low-latitude $\mathrm{CHs}$ from 1973 to present. We find that the peak of low-latitude CHs in the late declining phase of Solar Cycle 24 was only one half of the corresponding cycle peak in the previous Solar Cycles 21, 22, and 23. This behavior is reminiscent of the relative heights of the corresponding sunspot cycles and suggests that the amount of low-latitude $\mathrm{CHs}$ is related to the number of emerging active regions, being produced during their decay.

Acknowledgements We acknowledge the financial support by the Academy of Finland to the ReSoLVE Center of Excellence (Project 307411) and to the PRediction of SPace climate and its Effects in ClimaTe 
(PROSPECT, Project 321440) research project. MacIntosh Archive (McA) is constructed by NOAA National Center for Environmental Information (https://www.ngdc.noaa.gov/stp/space-weather/solar-data/solarimagery/composites/synoptic-maps/mc-intosh/ptmc_level3/).

Funding Note Open access funding provided by University of Oulu including Oulu University Hospital.

Disclosure of Potential Conflicts of Interest The authors declare that they have no conflicts of interest.

Publisher's Note Springer Nature remains neutral with regard to jurisdictional claims in published maps and institutional affiliations.

Open Access This article is licensed under a Creative Commons Attribution 4.0 International License, which permits use, sharing, adaptation, distribution and reproduction in any medium or format, as long as you give appropriate credit to the original author(s) and the source, provide a link to the Creative Commons licence, and indicate if changes were made. The images or other third party material in this article are included in the article's Creative Commons licence, unless indicated otherwise in a credit line to the material. If material is not included in the article's Creative Commons licence and your intended use is not permitted by statutory regulation or exceeds the permitted use, you will need to obtain permission directly from the copyright holder. To view a copy of this licence, visit http://creativecommons.org/licenses/by/4.0/.

\section{References}

Benevolenskaya, E.E., Kosovichev, A.G., Scherrer, P.H.: 2001, Detection of high-latitude waves of solar coronal activity in extreme-ultraviolet data from the Solar and Heliospheric Observatory EUV Imaging Telescope. Astrophys. J. 554, L107. DOI.

Caplan, R.M., Downs, C., Linker, J.A.: 2016, Synchronic coronal hole mapping using multi-instrument EUV images: data preparation and detection method. Astrophys. J. 823(1), 53. DOI.

Chapman, S.A.: 2007, PhD thesis. University of Central Lancashire Preston, UK.

Chapman, S.A., Bromage, B.J.I.: 2002, Variation of coronal hole area from solar minimum to maximum using EUV spectroscopic data from SOHO-CDS (preliminary results). In: Wilson, A. (ed.) From Solar Min to Max: Half a Solar Cycle with SOHO, ESA SP-508, 383. ADS.

de Toma, G.: 2011, Evolution of coronal holes and implications for high-speed solar wind during the minimum between Cycles 23 and 24. Solar Phys. 274(1), 195. DOI.

Gibson, S.E., Webb, D., Hewins, I.M., McFadden, R.H., Emery, B.A., Denig, W., McIntosh, P.S.: 2016, Beyond sunspots: studies using the mcintosh archive of global solar magnetic field patterns. Proc. Int. Astron. Union 12(S328), 93. DOI.

Hamada, A., Asikainen, T., Mursula, K.: 2019, New homogeneous dataset of solar EUV synoptic maps from SOHO/EIT and SDO/AIA. Solar Phys. 295(1), 2. DOI. ISBN 1573-093X.

Hamada, A., Asikainen, T., Virtanen, I., Mursula, K.: 2018, Automated identification of coronal holes from synoptic EUV maps. Solar Phys. 293(4), 71. DOI. ISBN 0038-0938.

Harrison, R.A.: 1997, EUV blinkers: the significance of variations in the extreme ultraviolet quiet Sun. Solar Phys. 175(2), 467. DOI. ISBN 1573-093X.

Harvey, K.L., Recely, F.: 2002, Polar coronal holes during Cycles 22 and 23. Solar Phys. 211(1/2), 31. DOI.

Heinemann, S.G., Temmer, M., Heinemann, N., Dissauer, K., Samara, E., Jerčić, V., Hofmeister, S.J., Veronig, A.M.: 2019, Statistical analysis and catalog of non-polar coronal holes covering the sdo-era using catch. Solar Phys. 294(10), 144. DOI. ISBN 1573-093X.

Hess Webber, S.A., Karna, N., Pesnell, W.D., Kirk, M.S.: 2014, Areas of polar coronal holes from 1996 through 2010. Solar Phys. 289, 4047. DOI.

Illarionov, E., Kosovichev, A., Tlatov, A.: 2020, Machine-learning approach to identification of coronal holes in solar disk images and synoptic maps. arXiv e-prints, arXiv. ADS.

Karna, N., Hess Webber, S.A., Pesnell, W.D.: 2014, Using polar coronal hole area measurements to determine the solar polar magnetic field reversal in Solar Cycle 24. Solar Phys. 289(9), 3381. DOI.

Kirk, M.S., Pesnell, W.D., Young, C.A., Hess Webber, S.A.: 2009, Automated detection of EUV polar coronal holes during Solar Cycle 23. Solar Phys. 257, 99. DOI.

Krista, L.D., Gallagher, P.T.: 2009, Automated coronal hole detection using local intensity thresholding techniques. Solar Phys. 256(1), 87. DOI.

Liewer, P.C., Qiu, J., Lindsey, C.: 2017, Comparison of helioseismic far-side active region detections with STEREO far-side EUV observations of solar activity. Solar Phys. 292(10), 146. DOI. 
Lowder, C., Jiong, Q., Leamon, R.: 2017, Coronal holes and open magnetic flux over Cycles 23 and 24. Solar Phys. 292(1), 18. DOI.

Lowder, C., Qiu, J., Leamon, R., Liu, Y.: 2014, Measurements of EUV coronal holes and open magnetic flux. Astrophys. J. 783(2), 142. DOI.

Lukianova, R., Mursula, K.: 2011, Changed relation between sunspot numbers, solar UV/EUV radiation and tsi during the declining phase of solar cycle 23. J. Atmos. Solar-Terr. Phys. 73(2), 235. Space Climate. DOI.

Madjarska, M.S., Wiegelmann, T.: 2009, Coronal hole boundaries evolution at small scales - I. EIT $195 \AA$ and TRACE $171 \AA ̊$ view. Astron. Astrophys. 503(3), 991. DOI.

Moses, D., Clette, F., Delaboudinière, J.-P., Artzner, G.E., Bougnet, M., Brunaud, J., Carabetian, C., Gabriel, A.H., Hochedez, J.-F., Millier, F., Song, X.Y., Au, B., Dere, K.P., Howard, R.A., Kreplin, R., Michels, D.J., Defise, J.-M., Jamar, C., Rochus, P., Chauvineau, J.P., Marioge, J.P., Catura, R.C., Lemen, J.R., Shing, L., Stern, R.A., Gurman, J.B., Neupert, W.M., Newmark, J.S., Thompson, B., Maucherat, A., Portier-Fozzani, F., Berghmans, D., Cugnon, P., Van Dessel, E.L., Gabryl, J.R.: 1997, EIT observations of the extreme ultraviolet Sun. Solar Phys. 175, 571. DOI.

Petkaki, P., Del Zanna, G., Mason, H.E., Bradshaw, S.J.: 2012, SDO AIA and EVE observations and modelling of solar flare loops. Astron. Astrophys. 547, A25. DOI.

Phillips, K.J.H.: 1995, Guide to the Sun, Cambridge University Press, Cambridge, 386. ISBN 052139788X.

Scholl, I.F., Habbal, S.R.: 2008, Automatic detection and classification of coronal holes and filaments based on EUV and magnetogram observations of the solar disk. Solar Phys. 248(2), 425. DOI.

Schrijver, C.J., McMullen, R.A.: 2000, A case for resonant scattering in the quiet solar corona in extremeultraviolet lines with high oscillator strengths. Astrophys. J. 531(2), 1121. DOI.

Suresh, S.: 2013, Framework for near real time feature detection from the atmospheric imaging assembly images of the solar dynamics observatory. $\mathrm{PhD}$ thesis.

Tlatov, A., Tavastsherna, K., Vasil'eva, V.: 2014, Coronal holes in Solar Cycles 21 to 23. Solar Phys. 289(April 2013), 1349. DOI.

Tousey, R., Sandlin, G.D., Purcell, J.D.: 1968, On some aspects of EUV spectroheliograms. In: Kiepenheuer, K.O. (ed.) Structure and Development of Solar Active Regions, IAU Symp. 35, 411.

Verbeeck, C., Delouille, V., Mampaey, B., De Visscher, R.: 2014, The spoca-suite: software for extraction, characterization, and tracking of active regions and coronal holes on euv images. Astron. Astrophys. 561, A29. DOI.

Virtanen, I., Mursula, K.: 2016, Photospheric and coronal magnetic fields in six magnetographs I. Consistent evolution of the bashful ballerina. Astron. Astrophys. 591, A78. DOI.

Virtanen, I.I., Koskela, J.S., Mursula, K.: 2020, Abrupt shrinking of solar corona in the late 1990s. Astrophys. J. 889(2), L28. DOI.

Waldmeier, M.: 1957, Die Sonnenkorona II, Verlag Birkhäuser, Basel.

Zar, J.H.: 2014, Spearman rank correlation: overview. In: Wiley StatsRef: Statistics Reference Online, Wiley, Chichester. DOI. 